\title{
Heavy Metals Uptake by Aerial Biomass and Grain of Soybean
}

\author{
Ivica Kisic, Aleksandra Jurisic, Hana Mesic and Sanja Mesic \\ University of Zagreb Faculty of Agriculture, Croatian Environment Agency, INA d.d.
}

Croatia

\section{Introduction}

All phases of crude oil exploitation, starting from drilling works to the construction of underground pipeline networks, including transport, processing and storage, are causing interventions and procedures hazardous to the environment. Although modern technical solutions and materials are used in all segments of petroleum industry warrant a high degree of safety, the occurrence of various incidents is unfortunately not fully excluded. Incidents leading to pipeline spillage and crude oil contamination of the environment constitute a hazard to natural resources, primarily soil and water, and depending on their severity can jeopardize, for a shorter or longer time, the intended use of land on which the incident occurred, namely make it unsuitable for plant production. Petroleum and gas fields are located in Pannonian area where cultivation of agricultural crops is dominated and among them the most represented are corn, wheat and soybean.

On the other side, these (petroleum and gas) activities generate waste - drilling fluids which contain different chemical compounds, some of which are ecologically hazardous (hydrocarbons), or toxic substances (heavy metals). As already mentioned, drilling fluids contain increased levels of some heavy metals (barium, zinc and mercury), so their possible application as liming material involves the risk of heavy metal accumulation in soil and plants (Agbogidi et al., 2007). To assess the extent to which such material may be useful or harmful to soil, and thereby to plants grown on it, since pollutants enter the animal and human food chain via soil and plants, investigations into this problem were undertaken (Nelson et al., 1984; Lengrand et al., 2005; Kabata-Pendias \& Mukherjee, 2007).

\section{Goals of investigation}

The research objectives were to investigate the possibility of:

- Effects of different concentrations of crude oil and drilling fluids on the uptake of heavy metals in aerial biomass or grain of soybean, maize and winter wheat,

- Calculate enrichment coefficient for some parts of aerial biomass or grain of investigated crops.

\section{Materials and methods}

The trial was set up on Luvisols (FAO, 2006) and located near Popovaca (N 45 31'49" - E $\left.16^{\circ} 34^{\prime} 48^{\prime \prime}\right)$ in the Pannonian agricultural part of Croatia. The total trial field covered 1.25 
hectare, the size of each trial plot was $96 \mathrm{~m}^{2}$ (6.0 $\mathrm{m} \times 4.0 \mathrm{~m} \times 4$ replications). At the beginning of investigations in May 2006, before preparing the seedbed layer, crude oil and drilling fluids were applied on the soil surface. The seedbed was prepared using a tractor-mounted rototiller to the depth of $25 \mathrm{~cm}$. Experiment was set up as a randomized complete block design with four replications of each of the following treatments:

I. Control (unamended soil);

II. Soil contaminated by crude oil $-8 \mathrm{~L} \mathrm{~m}^{-2}$;

III. Soil contaminated by crude oil $-4 \mathrm{~L} \mathrm{~m}^{-2}$;

IV. Soil contaminated by crude oil $-2 \mathrm{~L} \mathrm{~m}^{-2}$;

V. Soil contaminated by drilling fluids $-30 \mathrm{~kg} \mathrm{~m}^{-2}$;

VI. Soil contaminated by drilling fluids $-20 \mathrm{~kg} \mathrm{~m}^{-2}$, and

VII. Soil contaminated by drilling fluids $-10 \mathrm{~kg} \mathrm{~m}^{-2}$.

Soybean (Glycine max L. Merr), Maize (Zea mays L.) and Winter Wheat (Triticum aestivum L.) were grown on the trial field. Composite soil samples $(0-25 \mathrm{~cm})$, in four replications, were taken before crops were sown. Composite plant samples (aerial biomass and grain) were taken after crop harvest. The observed results were analyzed using ANOVA analysis, SAS Institute 9.1.3. The significance level was set at $5 \%$ in all statistical tests. Methods used to determine the studied parameters are given in Table 1.

\begin{tabular}{|l|l|}
\hline Analysis & Method \\
\hline $\begin{array}{l}\text { Preparation of soil samples for physical and chemical } \\
\text { analyses }\end{array}$ & ISO 11464:2004 \\
\hline $\begin{array}{l}\text { Determination of particle size distribution in mineral soil } \\
\text { material - Method by sieving and sedimentation }\end{array}$ & ISO 11277:2004 \\
\hline $\begin{array}{l}\text { Determination of organic (TOC/OM) and total carbon (TC) } \\
\text { after dry combustion (elementary analysis) }\end{array}$ & ISO 10694:2004 \\
\hline Soil organic matter (SOM) & $\begin{array}{l}\text { Tjurin (wet digestion) - } \\
\text { titrimetric }\end{array}$ \\
\hline Determination of $\mathrm{pH}$ in $\mathrm{CaCl},(1: 2.5(\mathrm{w} / \mathrm{v}))$ & ISO 10390:2004 \\
\hline Extraction of trace elements soluble in aqua regia & ISO 11466:2004 \\
\hline Determination of $\mathrm{Zn}, \mathrm{Pb}, \mathrm{Cd}, \mathrm{Co}, \mathrm{Ni}, \mathrm{Cr}$ and Cu using AAS & ISO 11047:2004 \\
\hline Determination of As, Ba, Mo, $\mathrm{V}$ and $\mathrm{Hg}$ using ICP-MS & $\begin{array}{l}\text { ISO 11885:1998 \& } \\
\text { ISO/DIS 22036:2006 }\end{array}$ \\
\hline
\end{tabular}

Table 1. Methods used in investigations

\section{Results}

Particle size distribution of the studied soil is shown in Table 2. Major physical and chemical characteristics of crude oil and water-based drilling fluids (muds) applied in the trial are given in Tables 3 and 4. 


\begin{tabular}{|c|c|c|c|c|c|c|c|}
\hline \multirow[b]{2}{*}{ Depth, } & \multirow[b]{2}{*}{ Soil } & \multicolumn{5}{|c|}{ Particle size distribution, $\%$} & \multirow[b]{2}{*}{ Texture } \\
\hline & & Coarse & Fine sand & Coarse silt & Fine silt & Clay & \\
\hline $\mathrm{cm}$ & horizon & & $\begin{array}{c}0.2-0.05 \\
\mathrm{~mm}\end{array}$ & $\begin{array}{c}0.05-0.02 \\
\mathrm{~mm}\end{array}$ & $\begin{array}{c}0.02-0.002 \\
\mathrm{~mm}\end{array}$ & $\begin{array}{c}<0.002 \\
\mathrm{~mm}\end{array}$ & class \\
\hline $0-15$ & $\mathrm{Ap}+\mathrm{Eg}$ & 2.0 & 5.40 & 46.25 & 27.75 & 18.60 & Silty loam \\
\hline $15-30$ & $\mathrm{Eg}+\mathrm{Btg}$ & 1.5 & 6.40 & 41.80 & 30.00 & 20.30 & Silty loam \\
\hline $30-45$ & Btg & 0.5 & 4.30 & 43.25 & 29.80 & 22.15 & $\begin{array}{c}\text { Silty clay } \\
\text { loam }\end{array}$ \\
\hline
\end{tabular}

Table 2. Particle size distribution of Luvisols

\begin{tabular}{|c|c|c|c|c|c|c|c|c|c|}
\hline \multicolumn{3}{|c|}{ Components of crude oil } & \multicolumn{2}{|c|}{ Sum, vol. \% } & \multicolumn{3}{|c|}{$\begin{array}{c}\text { Density at } \\
15^{\circ} \mathrm{C}, \mathrm{g} \mathrm{cm}^{-3}\end{array}$} & \multicolumn{2}{|c|}{$\begin{array}{c}\text { Viscosity at } \\
37.8^{\circ} \mathrm{C} \mathrm{mm}^{2} \mathrm{~s}^{-1}\end{array}$} \\
\hline \multirow{9}{*}{\multicolumn{3}{|c|}{$\begin{array}{l}\text { Light gasoline } \\
\text { Light gasoline + heavy gasoline } \\
\text { Kerosene } \\
\text { Gas oil } \\
\text { Low viscosity lubricant oil } \\
\text { Medium viscosity lubricant oil } \\
\text { High viscosity lubricant oil } \\
\text { Residue } \\
\text { Loss }\end{array}$}} & \multirow{9}{*}{\multicolumn{2}{|c|}{$\begin{array}{c}6.53 \\
32.00 \\
10.40 \\
17.57 \\
12.08 \\
6.75\end{array}$}} & \multicolumn{3}{|c|}{0.69} & \multirow{9}{*}{\multicolumn{2}{|c|}{$\begin{array}{c}7.5-20.6 \\
20.6-43 \\
>43\end{array}$}} \\
\hline & & & & & \multirow{8}{*}{\multicolumn{3}{|c|}{$\begin{array}{r}0.75 \\
0.82 \\
0.85 \\
0.86-0 . \\
0.89-0 .\end{array}$}} & & \\
\hline & & & & & & & & & \\
\hline & & & & & & & & & \\
\hline & & & & & & & & & \\
\hline & & & & & & & & & \\
\hline & & & & & & & & & \\
\hline & & & & & & & & & \\
\hline & & & & & & & & & \\
\hline $\begin{array}{c}\text { Water, } \\
\%\end{array}$ & $\begin{array}{c}\text { Total } \\
\text { sulphur, } \\
\%\end{array}$ & $\begin{array}{c}\text { Viscosity, } \\
30^{\circ} \mathrm{C}, \mathrm{mPa}\end{array}$ & $\begin{array}{c}\text { Viscosity, } \\
30^{\circ} \mathrm{C} \\
\mathrm{mm}^{2}\end{array}$ & \begin{tabular}{|c|} 
Pour \\
point, \\
${ }^{\circ} \mathrm{C}$
\end{tabular} & $\begin{array}{c}\text { Coke, } \\
\%\end{array}$ & $\begin{array}{c}\text { Ash, } \\
\%\end{array}$ & $\begin{array}{c}\text { Paraffins, } \\
\%\end{array}$ & $\begin{array}{c}\text { Asfalten, } \\
\%\end{array}$ & $\begin{array}{c}\text { Total } \\
\text { nitrogen, } \\
\%\end{array}$ \\
\hline 0.08 & 0.433 & 5.56 & 6.65 & -8 & 2.193 & 0.004 & 5.57 & 0.68 & 0.439 \\
\hline
\end{tabular}

Table 3. Some characteristics of crude oil applied in the trial

\begin{tabular}{|c|c|c|c|c|c|c|c|c|c|c|c|c|c|}
\hline & $\mathrm{Cd}$ & $\mathrm{Hg}$ & $\mathrm{Pb}$ & $\mathrm{As}$ & $\mathrm{Ni}$ & $\mathrm{Cu}$ & $\mathrm{Cr}$ & $\mathrm{Zn}$ & $\mathrm{Ba}$ & $\mathrm{V}$ & $\mathrm{Co}$ & $\mathrm{Mo}$ & $\mathrm{Ca}$ \\
\hline Minimum & 0.96 & 0.34 & 89 & 6.9 & 21.2 & 23 & 27 & 116 & 163 & 84 & 5.8 & 2.3 & 10000 \\
\hline Maximum & 1.03 & 37.70 & 209 & 9.0 & 57.5 & 188 & 54 & 755 & 2333 & 132 & 12.0 & 5.2 & 156000 \\
\hline Average & 1.01 & 20.84 & 137 & 8.2 & 35.6 & 79 & 36 & 437 & 1602 & 95 & 8.4 & 4.0 & 103000 \\
\hline
\end{tabular}

Table 4. Concentrations of measured elements in drilling fluids applied in the trial, $\mathrm{mg} \mathrm{kg}^{-1}$

\subsection{Changes in soil $\mathrm{pH}$, soil organic matter, total carbon and nitrogen}

Research results show marked and expected heterogeneity of the studied parameters. In trial treatments where drilling fluids were applied (V, VI and VII) soil $\mathrm{pH}$ was significantly higher compared to other treatments (Table 5). This was expected because drilling fluids are very rich with $\mathrm{CaCO}_{3}, \mathrm{CaO}, \mathrm{Ca}(\mathrm{OH})_{2}, \mathrm{Ca}(\mathrm{SO})_{4}$ and other calcium compounds. In treatments where crude oil was applied, soil $\mathrm{pH}$ was not significantly different compared to the control treatment. These findings indicate that crude oil had no influence on the changes in soil $\mathrm{pH}$. A highly significant difference in SOM concentrations compared to all other treatments was determined in the treatment where the largest amount of crude oil was applied (treatment II). In the control treatment and in treatment with drilling fluids, SOM is mainly composed 
of humic compounds and plant debris. In the crude oil contaminated soils, part of the "SOM" is in fact carbon hydrides from crude oil.

Changes in total carbon follow trends and statistical differences that are almost identical to SOM. Compared to the control treatment significantly higher carbon content was recorded in treatments where crude oil was applied (treatments II, III and IV) as well as in treatment with the highest dose of drilling fluids (treatment V). Significantly higher nitrogen content compared to the control treatment, was determined in treatments IV and V. No significant differences in nitrogen content were determined in other treatments.

\begin{tabular}{|l|c|c|c|c|c|c|c|}
\hline \multicolumn{1}{|c|}{ Treatment } & I & II & III & IV & V & VI & VII \\
\hline Soil $\mathrm{pH}$ & $5.34 \mathrm{~b}^{*}$ & $5.41 \mathrm{~b}$ & $5.23 \mathrm{~b}$ & $5.26 \mathrm{~b}$ & $6.58 \mathrm{a}$ & $6.59 \mathrm{a}$ & $6.92 \mathrm{a}$ \\
\hline SOM, $\%$ & $1.83 \mathrm{bc}$ & $4.07 \mathrm{a}$ & $2.07 \mathrm{bc}$ & $1.30 \mathrm{c}$ & $2.07 \mathrm{bc}$ & $1.83 \mathrm{bc}$ & $2.53 \mathrm{~b}$ \\
\hline Carbon, $\%$ & $1.70 \mathrm{c}$ & $2.78 \mathrm{a}$ & $2.57 \mathrm{a}$ & $2.40 \mathrm{ba}$ & $2.42 \mathrm{ba}$ & $2.03 \mathrm{bc}$ & $1.99 \mathrm{bc}$ \\
\hline Nitrogen, $\%$ & $0.184 \mathrm{c}$ & $0.200 \mathrm{bac}$ & $0.212 \mathrm{ba}$ & $0.230 \mathrm{a}$ & $0.222 \mathrm{a}$ & $0.182 \mathrm{bc}$ & $0.197 \mathrm{bac}$ \\
\hline $\mathrm{C} / \mathrm{N}$ ratio & $9.24 \mathrm{f}$ & $13.90 \mathrm{a}$ & $12.12 \mathrm{~b}$ & $10.43 \mathrm{de}$ & $10.90 \mathrm{dc}$ & $11.15 \mathrm{c}$ & $10.10 \mathrm{fe}$ \\
\hline
\end{tabular}

* Values are means of 4 replicates. For each parameter, values in the same row followed by an identical letter are not significantly different according to Fisher's LSD test $(P \leq 0.05)$.

Table 5. Changes in soil $\mathrm{pH}$, soil organic matter, carbon and nitrogen

\subsection{Heavy metals in soil}

The aim of this chapter is to show the degree to which crude oil or drilling fluids affect changes in heavy metal concentrations in soil. Cadmium concentrations in soil were lower than $0.3 \mathrm{mg} \mathrm{kg}^{-1}$ (Table 6). Regardless of the applied material (crude oil or drilling fluids), the detected changes in lead, molybdenum, arsenic, nickel, cobalt, cooper, chromium and vanadium concentrations cannot be associated with the application of the mentioned materials. Compared to the control treatment and treatments were crude oil was applied, significantly higher mercury, zinc and barium concentrations were recorded in treatments with drilling fluids.

\begin{tabular}{|c|c|c|c|c|c|c|c|}
\hline Treatment & I & II & III & IV & V & VI & VII \\
\hline $\mathrm{Cd}$ & $<0.3$ & $<0.3$ & $<0.3$ & $<0.3$ & $<0.3$ & $<0.3$ & $<0.3$ \\
\hline $\mathrm{Hg}$ & $0.03 \mathrm{c}^{*}$ & $0.05 \mathrm{c}$ & $0.04 \mathrm{c}$ & $0.05 \mathrm{c}$ & $0.37 \mathrm{a}$ & $0.33 \mathrm{a}$ & $0.14 \mathrm{~b}$ \\
\hline $\mathrm{Pb}$ & $30 \mathrm{a}$ & $25 \mathrm{a}$ & $22 \mathrm{a}$ & $27 \mathrm{a}$ & $26 \mathrm{a}$ & $25 \mathrm{a}$ & $31 \mathrm{a}$ \\
\hline $\mathrm{Mo}$ & $0.40 \mathrm{a}$ & $0.34 \mathrm{a}$ & $0.33 \mathrm{a}$ & $0.33 \mathrm{a}$ & $0.47 \mathrm{a}$ & $0.34 \mathrm{a}$ & $0.31 \mathrm{a}$ \\
\hline $\mathrm{As}$ & $8 \mathrm{a}$ & $9 \mathrm{a}$ & $9 \mathrm{a}$ & $9 \mathrm{a}$ & $9 \mathrm{a}$ & $10 \mathrm{a}$ & $8 \mathrm{a}$ \\
\hline $\mathrm{Ni}$ & $20 \mathrm{a}$ & $20 \mathrm{a}$ & $20 \mathrm{a}$ & $20 \mathrm{a}$ & $20 \mathrm{a}$ & $22 \mathrm{a}$ & $21 \mathrm{a}$ \\
\hline $\mathrm{Co}$ & $12 \mathrm{a}$ & $12 \mathrm{a}$ & $12 \mathrm{a}$ & $13 \mathrm{a}$ & $11 \mathrm{a}$ & $12 \mathrm{a}$ & $11 \mathrm{a}$ \\
\hline $\mathrm{Cu}$ & $17 \mathrm{a}$ & $17 \mathrm{a}$ & $19 \mathrm{a}$ & $19 \mathrm{a}$ & $20 \mathrm{a}$ & $19 \mathrm{a}$ & $19 \mathrm{a}$ \\
\hline $\mathrm{Cr}$ & $26 \mathrm{a}$ & $25 \mathrm{a}$ & $25 \mathrm{a}$ & $27 \mathrm{a}$ & $26 \mathrm{a}$ & $27 \mathrm{a}$ & $28 \mathrm{a}$ \\
\hline $\mathrm{Zn}$ & $62 \mathrm{~b}$ & $62 \mathrm{~b}$ & $64 \mathrm{~b}$ & $65 \mathrm{ba}$ & $78 \mathrm{a}$ & $78 \mathrm{a}$ & $72 \mathrm{a}$ \\
\hline $\mathrm{Ba}$ & $96 \mathrm{c}$ & $93 \mathrm{c}$ & $92 \mathrm{c}$ & $91 \mathrm{c}$ & $160 \mathrm{ab}$ & $194 \mathrm{a}$ & $144 \mathrm{~b}$ \\
\hline $\mathrm{V}$ & $33 \mathrm{a}$ & $32 \mathrm{a}$ & $34 \mathrm{a}$ & $36 \mathrm{a}$ & $34 \mathrm{a}$ & $35 \mathrm{a}$ & $36 \mathrm{a}$ \\
\hline
\end{tabular}

* Values are means of 4 replicates. For each parameter, values in the same row followed by an identical letter are not significantly different according to Fisher's LSD test $(P \leq 0.05)$.

Table 6. Total heavy metal concentrations in soil ( $\mathrm{mg} \mathrm{kg}^{-1}$ soil) 


\subsection{Plant uptake of heavy metals}

Concentrations of heavy metals in aerial biomass and grain of soybean and other crops are presented in Table 7. Higher levels of cadmium, chromium, zinc and barium were recorded in soybean aerial biomass than in its grain almost in all treatments. Soybean grain contained more nickel and copper compared to its aerial biomass. Values determined for molybdenum show no regularity. Higher levels of molybdenum were determined in

\begin{tabular}{|c|c|c|c|c|c|c|c|c|}
\hline \multirow{2}{*}{\multicolumn{2}{|c|}{ Treatment }} & I & II & III & IV & $\overline{\mathrm{V}}$ & VI & VII \\
\hline & & \multicolumn{7}{|c|}{ Soybean } \\
\hline $\begin{array}{l}\text { aerial biomass } \\
\text { grain }\end{array}$ & $\mathrm{Cd}$ & $\begin{array}{l}0.34 b^{*} \\
0.12 b\end{array}$ & $\begin{array}{c}0.21 \mathrm{c} \\
0.14 \mathrm{ab}\end{array}$ & $\begin{array}{c}0.35 \mathrm{~b} \\
0.15 \mathrm{ab}\end{array}$ & $\begin{array}{l}0.41 \mathrm{a} \\
0.16 \mathrm{a}\end{array}$ & $\begin{array}{l}0.20 \mathrm{c} \\
0.01 \mathrm{c}\end{array}$ & $\begin{array}{c}0.15 \mathrm{~d} \\
0.14 \mathrm{ab}\end{array}$ & $\begin{array}{l}0.11 \mathrm{e} \\
0.16 \mathrm{a}\end{array}$ \\
\hline $\begin{array}{l}\text { aerial biomass } \\
\text { grain }\end{array}$ & Mo & $\begin{array}{l}0.65 \mathrm{e} \\
0.81 \mathrm{c}\end{array}$ & $\begin{array}{l}1.29 \mathrm{a} \\
0.69 \mathrm{~d}\end{array}$ & $\begin{array}{l}1.17 \mathrm{~b} \\
0.42 \mathrm{e}\end{array}$ & $\begin{array}{l}0.69 \mathrm{e} \\
0.65 \mathrm{~d}\end{array}$ & $\begin{array}{l}1.11 \mathrm{c} \\
1.16 \mathrm{~b}\end{array}$ & $\begin{array}{l}0.64 \mathrm{e} \\
1.09 \mathrm{~b}\end{array}$ & $\begin{array}{l}0.79 \mathrm{~d} \\
1.47 \mathrm{a}\end{array}$ \\
\hline $\begin{array}{l}\text { aerial biomass } \\
\text { grain }\end{array}$ & $\mathrm{Ni}$ & $\begin{array}{l}4.02 \mathrm{~d} \\
8.71 \mathrm{~cd}\end{array}$ & $\begin{array}{l}14.00 \mathrm{a} \\
10.00 \mathrm{~b}\end{array}$ & $\begin{array}{l}8.56 \mathrm{~b} \\
9.21 \mathrm{c}\end{array}$ & $\begin{array}{l}5.74 \mathrm{c} \\
11.6 \mathrm{a}\end{array}$ & $\begin{array}{l}5.56 \mathrm{c} \\
10.3 \mathrm{~b}\end{array}$ & \begin{tabular}{|c|}
$5.18 \mathrm{c}$ \\
$8.91 \mathrm{~cd}$
\end{tabular} & $\begin{array}{l}4.84 \mathrm{~cd} \\
8.34 \mathrm{~d}\end{array}$ \\
\hline $\begin{array}{l}\text { aerial biomass } \\
\text { grain }\end{array}$ & $\mathrm{Cu}$ & $\begin{array}{l}10.3 \mathrm{a} \\
19.4 \mathrm{~b}\end{array}$ & $\begin{array}{c}7.23 \mathrm{ed} \\
17.7 \mathrm{c}\end{array}$ & $\begin{array}{l}7.12 \mathrm{ed} \\
18.4 \mathrm{bc}\end{array}$ & $\begin{array}{l}7.77 \mathrm{c} \\
19.2 \mathrm{~b}\end{array}$ & $\begin{array}{l}8.78 \mathrm{~b} \\
22.1 \mathrm{a}\end{array}$ & $\begin{array}{l}6.96 \mathrm{e} \\
19.5 \mathrm{~b}\end{array}$ & $\begin{array}{l}7.53 \mathrm{~cd} \\
18.4 \mathrm{bc}\end{array}$ \\
\hline $\begin{array}{l}\text { aerial biomass } \\
\text { grain }\end{array}$ & $\mathrm{Cr}$ & $\begin{array}{l}2.34 \mathrm{e} \\
1.43 \mathrm{a}\end{array}$ & $\begin{array}{l}6.43 \mathrm{a} \\
1.41 \mathrm{a}\end{array}$ & \begin{tabular}{|l}
$3.81 \mathrm{~b}$ \\
$1.31 \mathrm{ab}$
\end{tabular} & $\begin{array}{l}3.18 \mathrm{c} \\
1.35 \mathrm{ab}\end{array}$ & $\begin{array}{l}2.99 \mathrm{~cd} \\
1.26 \mathrm{ab}\end{array}$ & $\begin{array}{l}3.14 \mathrm{c} \\
1.16 \mathrm{bc}\end{array}$ & $\begin{array}{c}2.65 \mathrm{ed} \\
0.99 \mathrm{c}\end{array}$ \\
\hline $\begin{array}{l}\text { aerial biomass } \\
\text { grain }\end{array}$ & $\mathrm{Zn}$ & $\begin{array}{l}94 \mathrm{c} \\
50 \mathrm{e}\end{array}$ & $\begin{array}{l}98 \mathrm{~b} \\
54 \mathrm{~d}\end{array}$ & $\begin{array}{l}98 \mathrm{~b} \\
74 \mathrm{a}\end{array}$ & $\begin{array}{c}109 a \\
59 c\end{array}$ & $\begin{array}{l}81 \mathrm{~d} \\
67 \mathrm{~b}\end{array}$ & $\begin{array}{l}60 \mathrm{e} \\
62 \mathrm{c}\end{array}$ & $\begin{array}{l}61 \mathrm{e} \\
50 \mathrm{e}\end{array}$ \\
\hline $\begin{array}{c}\text { aerial biomass } \\
\text { grain }\end{array}$ & $\mathrm{Ba}$ & $\begin{array}{c}55 \mathrm{~d} \\
5.08 \mathrm{c} \\
\end{array}$ & $\begin{array}{c}60 \mathrm{c} \\
5.99 \mathrm{~b} \\
\end{array}$ & $\begin{array}{c}76 \mathrm{~b} \\
6.86 \mathrm{a}\end{array}$ & $\begin{array}{c}88 \mathrm{a} \\
6.91 \mathrm{a}\end{array}$ & $\begin{array}{l}57 \mathrm{~cd} \\
5.10 \mathrm{c}\end{array}$ & $\begin{array}{c}56 \mathrm{~d} \\
4.47 \mathrm{~d}\end{array}$ & $\begin{array}{c}42 \mathrm{e} \\
3.69 \mathrm{e}\end{array}$ \\
\hline \multicolumn{9}{|c|}{ Maize } \\
\hline $\begin{array}{l}\text { aerial biomass } \\
\text { grain }\end{array}$ & $\mathrm{Cd}$ & $\begin{array}{l}0.13 \mathrm{~d} \\
0.09 \mathrm{~b}\end{array}$ & $\begin{array}{l}0.24 \mathrm{a} \\
0.09 \mathrm{~b}\end{array}$ & $\begin{array}{l}0.24 \mathrm{a} \\
0.05 \mathrm{~d}\end{array}$ & $\begin{array}{l}0.16 \mathrm{c} \\
0.07 \mathrm{c}\end{array}$ & $\begin{array}{l}0.13 \mathrm{~d} \\
0.09 \mathrm{~b}\end{array}$ & $\begin{array}{l}0.20 \mathrm{~b} \\
0.08 \mathrm{bc}\end{array}$ & $\begin{array}{l}0.13 \mathrm{~d} \\
0.12 \mathrm{a}\end{array}$ \\
\hline $\begin{array}{c}\text { aerial biomass } \\
\text { grain }\end{array}$ & Mo & $\begin{array}{l}0.65 \mathrm{f} \\
0.40 \mathrm{~d} \\
\end{array}$ & $\begin{array}{l}0.74 \mathrm{~d} \\
0.83 \mathrm{a}\end{array}$ & $\begin{array}{l}0.42 \mathrm{~g} \\
0.52 \mathrm{c}\end{array}$ & $\begin{array}{l}0.68 \mathrm{e} \\
0.87 \mathrm{a}\end{array}$ & $\begin{array}{l}1.01 \mathrm{~b} \\
0.53 \mathrm{c}\end{array}$ & $\begin{array}{l}1.14 \mathrm{a} \\
0.68 \mathrm{~b}\end{array}$ & $\begin{array}{l}0.98 \mathrm{c} \\
0.72 \mathrm{~b}\end{array}$ \\
\hline $\begin{array}{l}\text { aerial biomass } \\
\text { grain }\end{array}$ & $\mathrm{Ni}$ & $\begin{array}{l}4.19 \mathrm{f} \\
4.86 \mathrm{e}\end{array}$ & $\begin{array}{l}5.03 \mathrm{c} \\
5.44 \mathrm{a}\end{array}$ & $\begin{array}{l}4.95 \mathrm{~d} \\
4.48 \mathrm{f}\end{array}$ & $\begin{array}{l}4.47 \mathrm{e} \\
5.13 \mathrm{~b}\end{array}$ & $\begin{array}{l}4.96 \mathrm{~d} \\
4.93 \mathrm{~d} \\
\end{array}$ & $\begin{array}{l}5.84 \mathrm{a} \\
5.05 \mathrm{c}\end{array}$ & $\begin{array}{l}5.46 \mathrm{~b} \\
3.96 \mathrm{~g}\end{array}$ \\
\hline $\begin{array}{l}\text { aerial biomass } \\
\text { grain }\end{array}$ & $\mathrm{Cu}$ & $\begin{array}{l}19.9 \mathrm{e} \\
2.45 \mathrm{c}\end{array}$ & $\begin{array}{l}18.3 \mathrm{f} \\
2.50 \mathrm{c}\end{array}$ & $\begin{array}{l}20.7 \mathrm{~d} \\
2.09 \mathrm{~d}\end{array}$ & $\begin{array}{l}24.8 \mathrm{a} \\
2.42 \mathrm{c}\end{array}$ & $\begin{array}{l}23.1 \mathrm{~b} \\
2.68 \mathrm{~b}\end{array}$ & $\begin{array}{l}21.9 \mathrm{c} \\
2.76 \mathrm{a}\end{array}$ & $\begin{array}{l}17.1 \mathrm{~d} \\
2.11 \mathrm{~d}\end{array}$ \\
\hline $\begin{array}{l}\text { aerial biomass } \\
\text { grain }\end{array}$ & $\mathrm{Cr}$ & $\begin{array}{l}3.61 \mathrm{c} \\
1.73 \mathrm{a}\end{array}$ & $\begin{array}{l}5.00 \mathrm{a} \\
1.64 \mathrm{~b}\end{array}$ & $\begin{array}{l}4.07 \mathrm{~b} \\
1.38 \mathrm{e}\end{array}$ & $\begin{array}{l}3.72 \mathrm{c} \\
1.63 \mathrm{~b}\end{array}$ & $\begin{array}{l}3.28 \mathrm{~d} \\
1.55 \mathrm{c}\end{array}$ & $\begin{array}{c}3.94 \mathrm{~b} \\
1.69 \mathrm{ab}\end{array}$ & $\begin{array}{l}3.18 \mathrm{~d} \\
1.46 \mathrm{~d}\end{array}$ \\
\hline $\begin{array}{l}\text { aerial biomass } \\
\text { grain }\end{array}$ & $\mathrm{Zn}$ & $\begin{array}{l}80 \mathrm{~b} \\
21 \mathrm{~b}\end{array}$ & $\begin{array}{l}64 \mathrm{e} \\
21 \mathrm{~b}\end{array}$ & $\begin{array}{l}79 \mathrm{~b} \\
25 \mathrm{a}\end{array}$ & $\begin{array}{l}88 \mathrm{a} \\
22 \mathrm{~b}\end{array}$ & $\begin{array}{l}75 \mathrm{c} \\
21 \mathrm{~b}\end{array}$ & $\begin{array}{c}67 \mathrm{de} \\
24 \mathrm{a}\end{array}$ & $\begin{array}{l}69 \mathrm{~d} \\
20 \mathrm{~b}\end{array}$ \\
\hline $\begin{array}{l}\text { aerial biomass } \\
\text { grain }\end{array}$ & $\mathrm{Ba}$ & $\begin{array}{l}17.6 \mathrm{~d} \\
9.70 \mathrm{~b}\end{array}$ & $\begin{array}{l}20.0 \mathrm{~b} \\
10.40 \mathrm{a}\end{array}$ & $\begin{array}{l}22.0 \mathrm{a} \\
7.65 \mathrm{e}\end{array}$ & $\begin{array}{l}18.8 \mathrm{c} \\
2.28 \mathrm{f}\end{array}$ & $\begin{array}{l}17.1 \mathrm{~d} \\
9.42 \mathrm{c}\end{array}$ & $\begin{array}{l}13.6 \mathrm{e} \\
9.43 \mathrm{c}\end{array}$ & $\begin{array}{l}13.7 \mathrm{e} \\
8.74 \mathrm{~d}\end{array}$ \\
\hline $\begin{array}{l}\text { aerial biomass } \\
\text { grain }\end{array}$ & $\mathrm{V}$ & $\begin{array}{l}0.62 \mathrm{e} \\
3.98 \mathrm{a}\end{array}$ & $\begin{array}{l}0.79 \mathrm{~cd} \\
1.17 \mathrm{c}\end{array}$ & \begin{tabular}{|c|}
$0.75 \mathrm{~cd}$ \\
$1.78 \mathrm{~b}$
\end{tabular} & $\begin{array}{l}1.50 \mathrm{~b} \\
1.03 \mathrm{c}\end{array}$ & $\begin{array}{c}0.69 \mathrm{de} \\
0.63 \mathrm{~d}\end{array}$ & $\begin{array}{l}0.81 \mathrm{c} \\
0.50 \mathrm{~d}\end{array}$ & $\begin{array}{l}2.68 \mathrm{a} \\
0.60 \mathrm{~d}\end{array}$ \\
\hline \multicolumn{9}{|c|}{ Winter Wheat } \\
\hline $\begin{array}{l}\text { aerial biomass } \\
\text { grain }\end{array}$ & $\mathrm{Cd}$ & $\begin{array}{c}0.13 \mathrm{bc} \\
0.17 \mathrm{a}\end{array}$ & $\begin{array}{l}0.19 \mathrm{a} \\
0.13 \mathrm{~b}\end{array}$ & \begin{tabular}{|l|}
$0.17 \mathrm{a}$ \\
$0.09 \mathrm{~d}$
\end{tabular} & $\begin{array}{l}0.14 \mathrm{~b} \\
0.11 \mathrm{bc}\end{array}$ & $\begin{array}{l}0.13 b c \\
0.13 b\end{array}$ & $\begin{array}{l}0.12 \mathrm{c} \\
0.10 \mathrm{~cd}\end{array}$ & $\begin{array}{l}0.08 \mathrm{~d} \\
0.18 \mathrm{a}\end{array}$ \\
\hline $\begin{array}{l}\text { aerial biomass } \\
\text { grain }\end{array}$ & $\mathrm{Hg}$ & $\begin{array}{l}0.02 \mathrm{a} \\
0.01 \mathrm{a}\end{array}$ & $\begin{array}{l}0.01 \mathrm{a} \\
0.02 \mathrm{a}\end{array}$ & $\begin{array}{l}0.01 \mathrm{a} \\
0.01 \mathrm{a}\end{array}$ & $\begin{array}{l}0.01 \mathrm{a} \\
0.02 \mathrm{a}\end{array}$ & $\begin{array}{l}0.01 \mathrm{a} \\
0.01 \mathrm{a}\end{array}$ & $\begin{array}{l}0.01 \mathrm{a} \\
0.01 \mathrm{a}\end{array}$ & $\begin{array}{l}0.01 \mathrm{a} \\
0.01 \mathrm{a}\end{array}$ \\
\hline
\end{tabular}




\begin{tabular}{|c|c|c|c|c|c|c|c|c|}
\hline \multirow{2}{*}{\multicolumn{2}{|c|}{ Treatment }} & I & II & III & IV & V & VI & VII \\
\hline & & \multicolumn{7}{|c|}{ Winter Wheat } \\
\hline \multirow{2}{*}{$\begin{array}{l}\text { aerial biomass } \\
\text { grain }\end{array}$} & \multirow{2}{*}{ Mo } & $0.74 \mathrm{~d}$ & $1.17 \mathrm{c}$ & $1.51 \mathrm{a}$ & $1.42 \mathrm{ab}$ & $1.23 \mathrm{bc}$ & $1.24 \mathrm{bc}$ & $1.28 \mathrm{bc}$ \\
\hline & & $0.92 \mathrm{~cd}$ & $0.99 \mathrm{bc}$ & $1.14 \mathrm{~b}$ & $1.41 \mathrm{~b}$ & $0.90 \mathrm{~cd}$ & $0.91 \mathrm{~cd}$ & $0.72 \mathrm{~d}$ \\
\hline \multirow{2}{*}{$\begin{array}{l}\text { aerial biomass } \\
\text { grain }\end{array}$} & \multirow{2}{*}{$\mathrm{Ni}$} & $3.52 \mathrm{~d}$ & $2.87 \mathrm{e}$ & $3.59 \mathrm{~d}$ & $4.78 \mathrm{~b}$ & $5.14 \mathrm{a}$ & $5.29 \mathrm{a}$ & $4.39 c$ \\
\hline & & $5.15 \mathrm{~b}$ & $5.62 \mathrm{a}$ & $5.13 \mathrm{~b}$ & $4.78 \mathrm{de}$ & $4.92 \mathrm{~cd}$ & $5.11 \mathrm{bc}$ & $4.64 \mathrm{e}$ \\
\hline \multirow{2}{*}{$\begin{array}{c}\text { aerial biomass } \\
\text { grain }\end{array}$} & \multirow{2}{*}{$\mathrm{Cu}$} & $3.56 \mathrm{e}$ & $4.28 \mathrm{c}$ & $4.58 \mathrm{~b}$ & $4.73 \mathrm{~b}$ & $4.98 \mathrm{a}$ & $3.91 d$ & $3.54 \mathrm{e}$ \\
\hline & & $7.56 \mathrm{~d}$ & $8.21 \mathrm{bc}$ & $8.47 \mathrm{a}$ & $8.17 \mathrm{bc}$ & $7.98 \mathrm{c}$ & $8.29 \mathrm{ab}$ & $8.04 \mathrm{c}$ \\
\hline \multirow{2}{*}{$\begin{array}{l}\text { aerial biomass } \\
\text { grain }\end{array}$} & \multirow{2}{*}{$\mathrm{Cr}$} & $1.47 \mathrm{~d}$ & $1.23 \mathrm{e}$ & $2.58 \mathrm{c}$ & $2.61 \mathrm{c}$ & $2.84 \mathrm{ab}$ & $2.71 \mathrm{bc}$ & $2.96 \mathrm{a}$ \\
\hline & & $1.48 \mathrm{~d}$ & $1.53 \mathrm{~d}$ & $2.14 \mathrm{c}$ & $2.18 \mathrm{c}$ & $2.56 \mathrm{~b}$ & $2.72 \mathrm{a}$ & $2.69 \mathrm{a}$ \\
\hline \multirow{2}{*}{$\begin{array}{l}\text { aerial biomass } \\
\text { grain }\end{array}$} & \multirow{2}{*}{$\mathrm{Zn}$} & $34 \mathrm{e}$ & $38 \mathrm{~d}$ & $42 \mathrm{~b}$ & $35 \mathrm{e}$ & $39 \mathrm{~cd}$ & $48 \mathrm{a}$ & $41 \mathrm{bc}$ \\
\hline & & $49 \mathrm{~d}$ & $52 c$ & $51 \mathrm{~cd}$ & $55 \mathrm{~b}$ & $58 \mathrm{a}$ & $52 c$ & $56 \mathrm{ab}$ \\
\hline \multirow{2}{*}{$\begin{array}{c}\text { aerial biomas } \\
\text { grain }\end{array}$} & \multirow{2}{*}{$\mathrm{Ba}$} & $12.47 \mathrm{~b}$ & $13.28 \mathrm{a}$ & $12.78 \mathrm{~b}$ & $11.05 \mathrm{c}$ & $13.63 \mathrm{a}$ & $12.47 \mathrm{~b}$ & $11.23 \mathrm{c}$ \\
\hline & & $4.57 \mathrm{~d}$ & $5.12 \mathrm{c}$ & $5.89 \mathrm{a}$ & $4.73 \mathrm{~d}$ & $4.96 \mathrm{c}$ & $5.12 \mathrm{c}$ & $5.47 \mathrm{~b}$ \\
\hline
\end{tabular}

* Values are means of 4 replicates. For each parameter, values in the same row followed by an identical letter are not significantly different according to Fisher's LSD test $(P \leq 0.05)$.

Table 7. Total heavy metal concentrations in aerial biomass and grain of investigated crops (mg kg-1 dry matter)

aerial biomass in some treatments while in other treatments higher levels were found in grain. The grains of soybean and wheat contained more cadmium than grain of maize. The higher content of barium was determined in grain of maize than in grains of soybean and wheat. The highest copper content was determined in aerial biomass of maize. The highest copper content was determined in grain of soybean. Compared to the aerial biomass of wheat the aerial biomass of soybean and maize contained a higher content of chromium and zinc.

The higher content of molybdenum was determined in aerial biomass and grain of wheat than in aerial biomass and grain of other two crops. The grains of wheat and maize contained higher content of mercury compared to soybean grain.

\subsection{Enrichment Coefficients (EC)}

In various literature enrichment coefficient is also called enrichment ratio; translocation coefficient; transfer factor; soil-plant transfer coefficient; accumulation factor; phytoaccumulation factor; bioaccumulation factor; biological adsorption coefficient and uptake coefficient (Zgorelec, 2009). Enrichment coefficient was calculated with equation: EC $=C_{\text {aerial biomass or grain }} / C_{\text {soil, }}$ where $C_{\text {aerial biomass or grain }}$ and $C_{\text {soil }}$ represent the metal concentration (mass ratio) in the aerial biomass or grain and in the soil on dry weight basis $(\mathrm{mg} / \mathrm{kg}$ in DM), respectively. Malayeri et al., 2008 grouped plant species according to their heavy metal uptake capacities and sensitivity to metal pollution:

$\begin{array}{ll}\text { High accumulator plants } & \text { EC between } 1-10 \\ \text { Moderately accumulator plants } & \text { EC between } 0.1-1.0 \\ \text { Low accumulator plants } & \text { EC between } 0.01-0.1 \\ \text { Non accumulator plants } & \text { EC }<0.01\end{array}$

The highest enrichment coefficient for all investigated crops was molybdenum (Table 8). In all cases, enrichment coefficient of molybdenum was higher for biomass than for grain. 
According to the higher values of enrichment coefficient the next metal is zinc. However, in this case, content of zinc in maize and soybean is higher in biomass than in grain, while in winter wheat higher content of zinc in grain was determined. Investigated crops also had very high enrichment coefficient for cadmium. As for molybdenum, cadmium had higher content in aerial biomass than in grain. High content of copper was determined in maize aerial biomass, while for soybean and wheat higher content of copper was determined in grain than in biomass. Increased content of nickel was determined in biomass as well as in grain of all investigated crops. All other investigated metals and metalloids $(\mathrm{Pb}, \mathrm{Hg}, \mathrm{As}, \mathrm{Co}$, $\mathrm{Cr}, \mathrm{Ba}$ and $\mathrm{V}$ ) had very low enrichment coefficient.

\begin{tabular}{|c|c|c|c|c|c|c|c|c|}
\hline \multirow{2}{*}{\multicolumn{2}{|c|}{ Treatment }} & I & II & III & IV & $\mathbf{V}$ & VI & VII \\
\hline & & \multicolumn{7}{|c|}{ Soybean } \\
\hline \multirow{2}{*}{$\begin{array}{c}\text { aerial biomass } \\
\text { grain }\end{array}$} & \multirow{2}{*}{$\mathrm{Cd}$} & 1.1 & 0.7 & 1.2 & 1.4 & 0.7 & 0.5 & 0.4 \\
\hline & & 0.4 & 0.5 & 0.5 & 0.5 & 0.0 & 0.5 & 0.5 \\
\hline \multirow{2}{*}{$\begin{array}{c}\text { aerial biomass } \\
\text { grain }\end{array}$} & \multirow{2}{*}{ Mo } & 1.6 & 3.8 & 3.5 & 2.1 & 2.4 & 1.9 & 2.5 \\
\hline & & 2.0 & 2.0 & 1.3 & 2.0 & 2.5 & 3.2 & 4.7 \\
\hline \multirow{2}{*}{$\begin{array}{l}\text { aerial biomass } \\
\text { grain }\end{array}$} & \multirow{2}{*}{$\mathrm{Ni}$} & 0.2 & 0.7 & 0.4 & 0.3 & 0.3 & 0.2 & 0.2 \\
\hline & & 0.4 & 0.5 & 0.5 & 0.6 & 0.5 & 0.4 & 0.4 \\
\hline \multirow{2}{*}{$\begin{array}{l}\text { aerial biomass } \\
\text { grain }\end{array}$} & \multirow{2}{*}{$\mathrm{Cu}$} & 0.6 & 0.4 & 0.4 & 0.4 & 0.4 & 0.4 & 0.4 \\
\hline & & 1.1 & 1.0 & 1.0 & 1.0 & 1.1 & 1.0 & 1.0 \\
\hline \multirow{2}{*}{$\begin{array}{l}\text { aerial biomass } \\
\text { grain }\end{array}$} & \multirow{2}{*}{$\mathrm{Cr}$} & 0.1 & 0.3 & 0.2 & 0.1 & 0.1 & 0.1 & 0.1 \\
\hline & & 0.1 & 0.1 & 0.1 & 0.1 & 0.0 & 0.0 & 0.0 \\
\hline \multirow{2}{*}{$\begin{array}{l}\text { aerial biomass } \\
\text { grain }\end{array}$} & \multirow{2}{*}{$\mathrm{Zn}$} & 1.5 & 1.6 & 1.5 & 1.7 & 1.0 & 0.8 & 0.8 \\
\hline & & 0.8 & 0.9 & 1.2 & 0.9 & 0.9 & 0.8 & 0.7 \\
\hline \multirow{2}{*}{$\begin{array}{l}\text { aerial biomass } \\
\text { grain }\end{array}$} & \multirow{2}{*}{$\mathrm{Ba}$} & 0.6 & 0.6 & 0.8 & 1.0 & 0.4 & 0.3 & 0.3 \\
\hline & & 0.1 & 0.1 & 0.1 & 0.1 & 0.0 & 0.0 & 0.0 \\
\hline \multicolumn{9}{|c|}{ Maize } \\
\hline \multirow{2}{*}{$\begin{array}{l}\text { aerial biomass } \\
\text { grain }\end{array}$} & \multirow{2}{*}{$\mathrm{Cd}$} & 0.4 & 0.8 & 0.8 & 0.5 & 0.4 & 0.7 & 0.4 \\
\hline & & 0.3 & 0.3 & 0.2 & 0.2 & 0.3 & 0.3 & 0.4 \\
\hline \multirow{2}{*}{$\begin{array}{l}\text { aerial biomass } \\
\text { grain }\end{array}$} & \multirow{2}{*}{ Mo } & 1.6 & 2.2 & 1.3 & 2.1 & 2.1 & 3.4 & 3.2 \\
\hline & & 1.0 & 2.4 & 1.6 & 2.6 & 1.1 & 2.0 & 2.3 \\
\hline \multirow{2}{*}{$\begin{array}{l}\text { aerial biomass } \\
\text { grain }\end{array}$} & \multirow{2}{*}{$\mathrm{Ni}$} & 0.2 & 0.3 & 0.2 & 0.2 & 0.2 & 0.3 & 0.3 \\
\hline & & 0.2 & 0.3 & 0.2 & 0.3 & 0.2 & 0.2 & 0.2 \\
\hline \multirow{2}{*}{$\begin{array}{l}\text { aerial biomass } \\
\text { grain }\end{array}$} & \multirow{2}{*}{$\mathrm{Cu}$} & 1.2 & 1.1 & 1.1 & 1.3 & 1.2 & 1.2 & 0.9 \\
\hline & & 0.1 & 0.1 & 0.1 & 0.1 & 0.1 & 0.1 & 0.1 \\
\hline \multirow{2}{*}{$\begin{array}{l}\text { aerial biomass } \\
\text { grain }\end{array}$} & \multirow{2}{*}{$\mathrm{Cr}$} & 0.1 & 0.2 & 0.2 & 0.1 & 0.1 & 0.1 & 0.1 \\
\hline & & 0.1 & 0.1 & 0.1 & 0.1 & 0.1 & 0.1 & 0.1 \\
\hline \multirow{2}{*}{$\begin{array}{l}\text { aerial biomass } \\
\text { grain }\end{array}$} & & 1.3 & 1.0 & 1.2 & 1.4 & 1.0 & 0.9 & 1.0 \\
\hline & $\mathrm{Zn}$ & 0.3 & 0.3 & 0.4 & 0.3 & 0.3 & 0.3 & 0.3 \\
\hline aerial biomass & & 0.2 & 0.2 & 0.2 & 0.2 & 0.1 & 0.1 & 0.1 \\
\hline grain & Ва & 0.1 & 0.1 & 0.1 & 0.0 & 0.1 & 0.0 & 0.1 \\
\hline aerial biomass & & 0.0 & 0.0 & 0.0 & 0.0 & 0.0 & 0.0 & 0.1 \\
\hline grain & V & 0.1 & 0.0 & 0.1 & 0.0 & 0.0 & 0.0 & 0.0 \\
\hline
\end{tabular}




\begin{tabular}{|c|c|c|c|c|c|c|c|c|}
\hline \multirow{2}{*}{\multicolumn{2}{|c|}{ Treatment }} & I & II & III & IV & V & VI & VI \\
\hline & & \multicolumn{7}{|c|}{ Winter Wheat } \\
\hline \multirow{2}{*}{$\begin{array}{l}\text { aerial biomass } \\
\text { grain }\end{array}$} & \multirow{2}{*}{$\mathrm{Cd}$} & 0.4 & 0.6 & 0.6 & 0.5 & 0.4 & 0.4 & 0.3 \\
\hline & & 0.6 & 0.4 & 0.3 & 0.4 & 0.4 & 0.3 & 0.6 \\
\hline \multirow{2}{*}{$\begin{array}{l}\text { aerial biomass } \\
\text { grain }\end{array}$} & \multirow{2}{*}{$\mathrm{Hg}$} & 0.7 & 0.2 & 0.3 & 0.2 & 0.0 & 0.0 & 0.1 \\
\hline & & 0.3 & 0.4 & 0.3 & 0.4 & 0.0 & 0.0 & 0.1 \\
\hline \multirow{2}{*}{$\begin{array}{l}\text { aerial biomass } \\
\text { grain }\end{array}$} & \multirow{2}{*}{ Mo } & 1.9 & 3.4 & 4.6 & 4.3 & 2.6 & 3.6 & 4.1 \\
\hline & & 2.3 & 2.9 & 3.5 & 4.3 & 1.9 & 2.7 & 2.3 \\
\hline \multirow{2}{*}{$\begin{array}{l}\text { aerial biomass } \\
\text { grain }\end{array}$} & \multirow{2}{*}{$\mathrm{Ni}$} & 0.2 & 0.1 & 0.2 & 0.2 & 0.3 & 0.2 & 0.2 \\
\hline & & 0.3 & 0.3 & 0.3 & 0.2 & 0.2 & 0.2 & 0.2 \\
\hline \multirow{2}{*}{$\begin{array}{l}\text { aerial biomass } \\
\text { grain }\end{array}$} & \multirow{2}{*}{$\mathrm{Cu}$} & 0.2 & 0.3 & 0.2 & 0.2 & 0.2 & 0.2 & 0.2 \\
\hline & & 0.4 & 0.5 & 0.4 & 0.4 & 0.4 & 0.4 & 0.4 \\
\hline \multirow{2}{*}{$\begin{array}{l}\text { aerial biomass } \\
\text { grain }\end{array}$} & \multirow{2}{*}{$\mathrm{Cr}$} & 0.1 & 0.0 & 0.1 & 0.1 & 0.1 & 0.1 & 0.1 \\
\hline & & 0.1 & 0.1 & 0.1 & 0.1 & 0.1 & 0.1 & 0.1 \\
\hline \multirow{2}{*}{$\begin{array}{l}\text { aerial biomass } \\
\text { grain }\end{array}$} & \multirow{2}{*}{$\mathrm{Zn}$} & 0.5 & 0.6 & 0.7 & 0.5 & 0.5 & 0.6 & 0.6 \\
\hline & & 0.8 & 0.8 & 0.8 & 0.8 & 0.7 & 0.7 & 0.8 \\
\hline \multirow{2}{*}{$\begin{array}{l}\text { aerial biomass } \\
\text { grain }\end{array}$} & \multirow{2}{*}{$\mathrm{Ba}$} & 0.1 & 0.1 & 0.1 & 0.1 & 0.1 & 0.1 & 0.1 \\
\hline & & 0.0 & 0.1 & 0.1 & 0.1 & 0.0 & 0.0 & 0.0 \\
\hline
\end{tabular}

Table 8. Enrichment coefficient for investigated crops

\section{Discussion}

In the presented study, the application of crude oil did not cause elevated levels of heavy metals in soil, but the application of drilling fluids resulted in higher mercury, zinc and barium concentrations. Increased zinc and barium concentrations in treatments involving drilling fluids are attributed to the use of zinc carbonate and barite $\left(\mathrm{BaSO}_{4}\right)$ in drilling (U.S. Congress, Office of Technology Assessment, 1992). Barium is insoluble, inert and non-toxic (Deuel, 2003), and therefore is not considered to be a great soil problem. Increased mercury concentrations in treatments where drilling fluids were applied are attributed to the source of drilling fluids. The reason why drilling fluids contain a larger amount of calcium compounds is its use as an additive for inhibiting corrosion of oil and gas pipes, or as an additive to increase drilling fluid density (Veil \& Dusseault, 2003; Kisic et al., 2009). Also, material with increased calcium compounds is usually added to bind fluids when repairing a pipe break or other incidents.

The problem of increased concentrations of some heavy metals caused by the application of crude oil-based fluids was pointed out by Nelson et al., 1984. When crude oil-based fluids were used as liming material, the expected positive changes in soil $\mathrm{pH}$ occurred (Deeley \& Canter, 1986).

As optimum soil reaction for the majority of cultivated plants is around the neutral $\mathrm{pH}$ value, liming is a desirable practice because under neutral to weakly alkaline conditions bioavailability of some heavy metals (except As, V, Mo, Co and Cr) decreases (Mathur et al., 1991; Dermatas \& Meng, 2003). This reduces the possibility of their translocation from roots into the plant itself (Bolan et al., 2003).

Levels of heavy metals detected in aerial biomass or grain of crops in all trial treatments are within the ranges determined in some other studies (Adriano, 2001; Kabata-Pendias \& Mikherjee, 2007). A number of authors (Darell et al., 1984; Khan et al., 2000; Lengrand et al., 
2005) report that soil $\mathrm{pH}$, electrical conductivity, mechanical composition, organic matter concentrations, crop type and total heavy metals concentrations have a decisive influence on the heavy metal uptake by plants.

\section{Conclusions}

In soybean and wheat grain more nickel and copper were determined compared to soybean and wheat biomass. Also, higher content of zinc in wheat grain compared to biomass was determined. In all other cases, higher concentrations of heavy metals in aerial biomass than in grain of investigated crops were determined.

Regarding other investigated crops soybean had the highest enrichment coefficient for cadmium, copper, nickel and zinc.

\section{References}

Adriano, D.C. (2001). Bioavailability of Trace metals, In: Trace Elements in Terrestrial Environments - Biogeochemistry, Bioavailability, and Risks of Metals, MaryAnn Brickner, (Ed.), 61-90, Springer-Verlag, ISBN 0-387-98678-2, New York

Agbogidi, O.M.; Eruotor, P.G.; Akparobi, S.O. \& Nnaji, G.U. (2007). Heavy Metal Concentrations of Maize (Zea mays L.) Grown in Soil Contaminated with Crude Oil. International Journal of Botany, Vol. 3, No. 4, 385-389, ISSN 1811-9700

Bolan, N.S.; Adriano, D.C.; Mani A.P. \& Duraisamy A. (2003). Immobilization and phytoavailability of cadmium in variable charge soils. II. Effect of lime compost. Plant and Soil, Vol. 251, No. 2, (April 2003) 187-198, ISSN 0032-079X (Print) 15735036 (Online)

Deeley, G.M. \& Canter, L.W. (1986). Distribution of Heavy Metals in Waste Drilling Fluids under Conditions of Changing pH. Journal of Environmental Quality, Vol. 15, No. 2, (April-June 1986) 108-112, Online ISSN: 1537-2537 Print ISSN: 0047-2425

Dermatas, D. \& Meng, X.G. (2003). Utilization of fly ash for stabilization/solidification of heavy metal contaminated soils. Engineering Geology, Vol. 70 (May 2003) 377-394, ISSN 0013-7952

Deuel, L.E. Jr. (2003). Should we be Concerned with Barium? Soil Quality Guidelines for Barite: Environmental Health and Human Health. Alberta Environment, Canada, http://environment.gov.ab.ca/info/library/6298.pdf

FAO (2006). World reference base for soil resources. A framework for international classification, correlation and communication, p. 145, Rome, Italy

Kabata-Pendias, A. \& Mukherjee, A.B. (2007). Biogeochemistry of Trace Elements, In: Trace Elements from Soil to Human, Agata Oelschläger, (Ed.), 85-450, Springer, 978-3-54032713-4 (Print) 978-3-540-32714-1 (Online), Berlin Heidelberg New York

Khan, A.G.; Kuek, C.; Chaudhry, T.M.; Koo, C.S. \& Hayes, W. (2000). Role of plants, mycorrhizae and phytochelators in heavy metal contaminated land remediation. Chemosphere, Vol. 41, No.1-2, (July 2000) 197-207, ISSN 0045-6535

Kisic, I.; Mesic, S.; Basic, F.; Brkic, V.; Mesic, M.; Durn, G.; Zgorelec, Z. \& Bertovic, L. (2009).

The effect of drilling fluids and crude oil on some chemical characteristics of soil and crops. Geoderma, Vol. 149, No. 3-4, (March 2009) 209-216, ISSN 0016-7061

Lengrand, P.; Turmel, M.C.; Sauvé, S. \& Courchesne, F. (2005). Speciation and bioavailability of trace metals $(\mathrm{Cd}, \mathrm{Cu}, \mathrm{Ni}, \mathrm{Pb}, \mathrm{Zn})$ in the rhizosphere of contaminated soils, $\mathrm{In}$ : 
Biogeochemistry of trace elements in the rhizosphere, Huang, P.M. \& Gobran, G.R. (Ed.), 261-300, Elsevier-Pergamon, ISBN 10: 0-444-51997-1, Oxford

Mathur, B.S.; Rana, N.K. \& Lal, S. (1991). Effect of rhizosphere application of lime on crop yield, soil properties, nutrient uptake and economics. Journal of the Indian Society of Soil Science, Vol. 39, No. 3, 523-529, Print ISSN: 0019-638X Online ISSN: 0974-0228

Malayeri B.E.; Chehregani A.; Yousefi N. \& Lorestani B. (2008). Identification of the hyper accumulator plants in copper and iron mine in Iran. Pakistan Journal of Biological Science, Vol. 11, No. 3, (February 2008) 490-492, ISSN 1028-8880

Nelson, D.W.; Liu, S.L. \& Sommers, L.E. (1984). Extractability and Plant uptake of Trace Elements from Drilling Fluids. Journal of Environmental Quality, Vol. 13, No. 4, (October-December 1984) 562-566, Online ISSN: 1537-2537 Print ISSN: 0047-2425

Veil, J.A. \& Dusseault, M.B. (2003). Evaluation of slurry injection technology for management of drilling wastes. US Department of Energy National Petroleum Technology Office, p. 110

Zgorelec Z. (2009). Phytoaccumulation of Metals and Metalloids from Soil Polluted by Coal Ash. p. 105. Dissertation. Faculty of Agriculture University of Zagreb

***(1992). U.S. Congress, Office of Technology Assessment: Managing Industrial Solid Wastes from manufacturing, Mining, Oil and Gas Production, and Utility Coal Combustion. Background paper, OTA-BP-O-82, p. 12

http://www.fas.org/ota/ reports/9225.pdf 


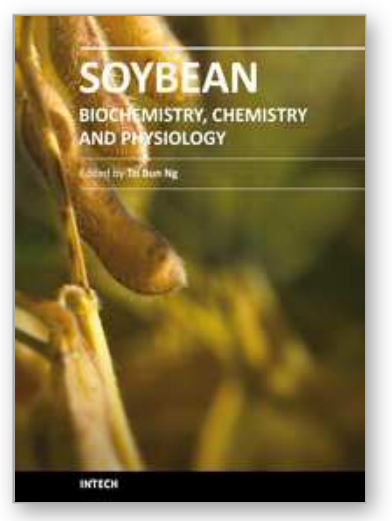

\section{Soybean - Biochemistry, Chemistry and Physiology \\ Edited by Prof. Tzi-Bun Ng}

ISBN 978-953-307-219-7

Hard cover, 642 pages

Publisher InTech

Published online 26, April, 2011

Published in print edition April, 2011

Soybean is an agricultural crop of tremendous economic importance. Soybean and food items derived from it form dietary components of numerous people, especially those living in the Orient. The health benefits of soybean have attracted the attention of nutritionists as well as common people.

\section{How to reference}

In order to correctly reference this scholarly work, feel free to copy and paste the following:

Ivica Kisic, Aleksandra Jurisic, Hana Mesic and Sanja Mesic (2011). Heavy Metals Uptake by Aerial Biomass and Grain of Soybean, Soybean - Biochemistry, Chemistry and Physiology, Prof. Tzi-Bun Ng (Ed.), ISBN: 978953-307-219-7, InTech, Available from: http://www.intechopen.com/books/soybean-biochemistry-chemistryand-physiology/heavy-metals-uptake-by-aerial-biomass-and-grain-of-soybean

\section{INTECH}

open science | open minds

\section{InTech Europe}

University Campus STeP Ri

Slavka Krautzeka 83/A

51000 Rijeka, Croatia

Phone: +385 (51) 770447

Fax: +385 (51) 686166

www.intechopen.com

\section{InTech China}

Unit 405, Office Block, Hotel Equatorial Shanghai

No.65, Yan An Road (West), Shanghai, 200040, China

中国上海市延安西路65号上海国际贵都大饭店办公楼405单元

Phone: +86-21-62489820

Fax: $+86-21-62489821$ 
(C) 2011 The Author(s). Licensee IntechOpen. This chapter is distributed under the terms of the Creative Commons Attribution-NonCommercialShareAlike-3.0 License, which permits use, distribution and reproduction for non-commercial purposes, provided the original is properly cited and derivative works building on this content are distributed under the same license. 